\title{
Factors associated with FEV1 decline in cystic fibrosis: analysis of the ECFS Patient Registry
}

\author{
Eitan Kerem', Laura Viviani², Anna Zolin², Stephanie MacNeill³, \\ Elpis Hatziagorou ${ }^{4}$, Helmut Ellemunter ${ }^{5}$, Pavel Drevinek ${ }^{6,7}$, Vincent Gulmans ${ }^{8}$, \\ Uros Krivec ${ }^{9}$ and Hanne Olesen ${ }^{10}$ on behalf of the ECFS Patient Registry \\ Steering Group \\ Affiliations: \\ 'Dept of Pediatrics and CF Center, Hadassah Hebrew University Hospital, Jerusalem, Israel. \\ ${ }^{2}$ Dept of Clinical Sciences and Community Health, University of Milan, Milan, Italy. \\ ${ }^{3}$ Dept of Occupational and Environmental Medicine, National Heart and Lung Institute, Imperial College \\ London, London, UK. \\ 43rd Dept of Paediatrics, CF Centre of Aristotelian University of Thessaloniki, Hippokration Hospital, \\ Thessaloniki, Greece. \\ ${ }^{5}$ Dept of Pediatrics, Univ.-Klinik für Pädiatrie III, Cystic Fibrosis Center, Medical University Innsbruck, Austria. \\ ${ }^{6}$ Dept of Paediatrics, 2nd Faculty of Medicine, Charles University and University Hospital Motol, Prague, and \\ ${ }^{7}$ Dept of Medical Microbiology, 2nd Faculty of Medicine, Charles University and University Hospital Motol, \\ Prague, Czech Republic. \\ ${ }^{8}$ Dutch Cystic Fibrosis Foundation (NCFS), Baarn, The Netherlands. \\ 'Unit for Pulmonary Diseases, University Children's Hospital, Ljubljana, Slovenia. \\ ${ }^{10}$ Dept of Paediatrics, Aarhus University Hospital, Aarhus, Denmark.
}

Correspondence: E. Kerem, Dept of Pediatrics and CF Center, Hadassah Hebrew University Hospital, MountScopus POB 24035, Jerusalem, 91240, Israel. E-mail: keremahadassah.org.il

ABSTRACT Pulmonary insufficiency is the main cause of death in cystic fibrosis (CF). We analysed forced expiratory volume in $1 \mathrm{~s}$ (FEV1) data of 14732 patients registered in the European Cystic Fibrosis Society Patient Registry (ECFSPR) database in 2007. We used linear and logistic regressions to investigate associations between FEV1\% predicted and clinical outcomes.

Body mass index (BMI), chronic infection by Pseudomonas aeruginosa, pancreatic status and CF-related diabetes $(\mathrm{CFRD})$ showed a statistically significant (all $\mathrm{p}<0.0001$ ) and clinically relevant effect on FEV1\% pred after adjusting for age. Patients with a lower BMI experience a six-fold increased odds ratio (95\% CI 5.0-7.3) of having severe lung disease (FEV1 $<40 \%$ pred) compared to patients with normal BMI. Being chronically infected with $P$. aeruginosa increases the odds ratio of severe lung disease by 2.4 (95\% CI 2.0-2.7), and patients with pancreatic insufficiency experience a 2.0-fold increased odds ratio (95\% CI 1.6-2.5) of severe lung disease compared to pancreatic sufficient patients. Patients with CFRD have a 1.8-fold increased odds ratio (95\% CI 1.6-2.2) compared to patients not affected.

These potential risk factors for pulmonary disease in patients with CF are to some degree preventable or treatable. We emphasise the importance of their early identification through frequent routine tests, the implementation of infection control measures, and a timely initiation of relevant therapies.

@ERSpublications

Independent effect of nutrition, chronic Pseudomonas aeruginosa infection and CF-related diabetes on FEV1 in CF patients http://ow.ly/qhAXJ 


\section{Introduction}

Despite considerable improvement in median survival, cystic fibrosis (CF) remains a life-shortening disease with pulmonary insufficiency as the main cause of death. Several methods are used to assess pulmonary disease severity in CF, including spirometry, chest imaging and quality-of-life questionnaires. For prognosis, forced expiratory volume in $1 \mathrm{~s}$ (FEV1) compared to a reference population (FEV1\% predicted), is regarded as the best generally available measure for assessing CF lung disease [1]. FEV1 is currently still an influential driver for the definition of disease stage, for decisions on treatment $[1,2]$, for comparison between centres and countries [3-5], as a primary outcome in clinical studies $[6,7]$ and in the regulatory approval of respiratory therapies in $\mathrm{CF}[8]$.

The European Cystic Fibrosis Society Patient Registry (ECFSPR) collects data on an annual basis according to agreed definitions and formats of a common set of variables [9] from individual CF centres and national CF registries in Europe and neighbouring countries. Data collection in 2007 included $>20000$ CF patients from 16 countries [10]. Such data collection is a reflection of the reality of CF across Europe, offering a unique basis for epidemiological analyses due to its international nature. It offers the possibility to evaluate factors potentially associated with FEV1 in a population that shows a wide variability of both lung disease and potential risk factors. The aim of this study was to review the association between FEV1 and factors previously described in the literature as major risk factors of CF lung disease.

\section{Patients and methods}

We used data from patients with CF registered in the ECFSPR in 2007. Data were provided by the national CF registries of Belgium, Czech Republic, Denmark, France, Germany, Israel, the Netherlands and the UK and by individual CF centres from Austria, Bulgaria, Greece, Italy, Portugal and Slovenia.

We analysed the association of the following variables with FEV1: age at diagnosis, age at FEV1 measurement, sex, pancreatic status, chronic Pseudomonas aeruginosa infection, CF-related diabetes (CFRD), body mass index (BMI) and genotype. These variables had a satisfactory level of completeness in the ECFSPR database (table 1).

In this study, pancreatic insufficiency was defined as the use of enzymes and pancreatic sufficiency as no use of enzymes during 2007. Chronic infection by P. aeruginosa was defined according to the modified Leeds criteria and/or presence of antibodies [9] by the clinicians at each centre. The UK defined chronic infection as patients having three or more positive isolates during the previous 12 months. CFRD was identified as use of insulin during 2007. We investigated the effect of genotype by classifying alleles according to McKonE et al. [11]. We grouped the alleles as "severe" (belong to either class I, II or III), "mild" (belong either to class IV or V) and "unclassified" (could not be classified to any of the previous classes, including the alleles that belong to class "unknown" according to MCKone et al. [11]).

We then grouped the patients according to such genotypes in the following classes: severe/severe (i.e. both alleles belonging to class I, II or III), severe/mild (i.e. one allele belonging to class I, II or III and the other allele belonging to class IV or V), mild/mild (both alleles belonging to class IV or V) or unclassified (UN) (at least one of two alleles unclassified).

The ECFSPR collects the best FEV1 value performed over the year and the corresponding height and weight. Three countries make an exception: France reported the last FEV1 of the year, Germany the value closest to the patient's birthday, and the UK the measurement taken at the annual assessment. All FEV1 values in litres were transformed into per cent predicted (\% pred) according to WANG et al. [12] for children and HANKINSON et al. [13] for adults. We used Centers for Disease Control and Prevention references [14] for computation of BMI standard deviation scores (SDS) and we categorised them into SDS $\leqslant-2,-2<\operatorname{SDS}<2$ and SDS $\geqslant 2$.

\section{Statistical analysis}

We used linear and logistic regressions to investigate association between FEV1\% pred and age, age at diagnosis, sex, pancreatic status, chronic P. aeruginosa infection, CFRD, BMI and genotype. Further details on statistical analyses are provided in the online supplementary material.

This article has supplementary material available from www.erj.ersjournals.com

Received: Oct 172012 | Accepted after revision: April 092013 | First published online: April 182013

Support statement: The ECFSPR is supported by an unrestricted grant from Chiesi Farmaceutici S.p.A. and from IERFC (European Institute for Research in Cystic Fibrosis). None of the funding sources had a role in study design; in the collection, analysis, and interpretation of data; in writing or reviewing the paper; nor in the decision to submit the paper for publication. 


\section{TABLE 1 Clinical and demographic characteristics of the study population}

Patients $\mathbf{n}$

Participating countries

Austria

$90(0.6)$

Belgium ${ }^{\#}$

$757(5.1)$

Bulgaria

$23(0.2)$

Czech Republic

313 (2.1)

Denmark $^{\#}$

France $^{\#}$

Germany

Greece

320 (2.2)

Israel

$3422(23.2)$

$4133(28.0)$

$56(0.4)$

Italy

$390(2.6)$

The Netherlands ${ }^{\#}$

$632(4.3)$

Portugal

871 (5.9)

$78(0.5)$

Slovenia

$37(0.2)$

UK

3610 (24.5)

Male

7772 (52.8)

Age at FEV1 measurement years

Median (range)

$\geqslant 18$ years

Age at diagnosis months ${ }^{\pi_{0}+}$

$19.0(6-76.7)$

$8001(54.3)$

Median (range)

$\geqslant 18$ years

$7.2(0-73.3)$

$668(6.1)$

Genotype

Patients with DNA analysis

Severe/severe

Severe/mild

Mild/mild

At least one allele unclassified

$3613(92.4)$

$8624(63.3)$

$731(5.4)$

$27(0.2)$

$4231(31.1)$

Unknown alleles

$2344(8.6)$

BMI $\leqslant-2$ SDS

6-9 years

10-14 years

15-19 years

111 (5.1)

191 (6.7)

$316(11.4)$

$20-24$ years

25-29 years

30-34 years

$340(14.9)$

$35-39$ years

225 (13.3)

104 (9.3)

40-44 years

$\geqslant 45$ years

$65(8.0)$

$21(4.2)$

Pancreatic status $\# \#$

Pancreatic insufficient

18 (4.3)

Chronic Pseudomonas aeruginosa infection ${ }^{\text {शा }}$

Infected patients

$11567(86.7)$

CFRD ${ }^{++}$

$0-9$ years

10-19 years

20-29 years

$\geqslant 30$ years

3631 (37.2)

FEV1 \% predicted

6-9 years

$10-14$ years

$15-19$ years

20-24 years

25-29 years

30-34 years

35-39 years

40-44 years

$\geqslant 45$ years

$14(0.7)$

$409(7.6)$

697 (18.2)

$619(22.7)$

$91.2(90.4-91.9)$

$86.5(85.7-87.2)$

$72.3(71.4-73.1)$

$63.0(62.0-64.0)$

$58.5(57.4-59.7)$

$56.4(55.0-57.8)$

$56.9(55.3-58.6)$

$55.5(53.4-57.6)$

$57.7(55.3-60.0)$

Data are presented as $\mathrm{n}(\%)$ or mean $(95 \% \mathrm{CI})$, unless otherwise stated. FEV1: forced expiratory volume in $1 \mathrm{~s}$; BMI: body mass index; SDS: standard deviation score; CFRD: cystic fibrosis-related diabetes. " : data sent by a national registry; ": data available for 10994 patients; ${ }^{+}$: for prenatal diagnoses, age at diagnosis was set to 0 months; ${ }^{\S}$ : alleles classified according to McKonE et al. [11]: severe lalleles that belong to either class I, II or III), mild (alleles that belong either to class IV or V) and unclassified (alleles that could not be classified into any of the previous classes); ${ }^{f}$ : data available for 14625 patients; ${ }^{\# \#}$ : data available for 13343 patients; "थ: data available for 9748 patients; ${ }^{++}$: data available for 14013 patients.

\section{Results}

\section{Study population}

Data referring to 20204 patients with CF seen in a CF clinic during 2007 were registered in the ECFSPR. The 4420 children aged $<6$ years were excluded from the analyses due to their unreliable ability to perform spirometry and to the lack of valid reference values for lung function. The 446 patients who had had a lung transplantation were excluded, as their FEV1 did not represent their disease stage (the UK registry gave 
information only on transplants performed during 2007, so patients from the UK that had had a lung transplant before 2007 are included). For an additional 606 (3.9\%) patients, FEV1 \% pred could not be computed because of missing values for FEV1 or height. Our final study population included 14732 patients (52.8\% males).

Table 1 describes the main demographic and clinical characteristics of the included patients. Information on genotype was available for $13613(92.4 \%)$ patients and $8.6 \%$ of the alleles remained unidentified after DNA analysis. When classified according to MCKone et al. [11], 63.3\% of patients carried two alleles belonging to class I or II or III, 5.4\% of patients carried one allele belonging to class I or II or III and the other allele belonging to class IV or V, and $0.2 \%$ of the patients carried two alleles belonging to class IV or $\mathrm{V}$. The remaining patients carried at least one allele that could not be classified. When classified according to F508del genotype, $86.7 \%$ of patients carried the F508del allele (48.3\% in homozygosity and $38.4 \%$ in compound heterozygosity). The proportion of patients with low BMI (SDS $\leqslant-2$ ) increased from children to young adults but decreased again in older patients ( $>30$ years of age). $86.7 \%$ of the patients had pancreatic insufficiency. Prevalence of chronic infection with $P$. aeruginosa gradually increased with age until it reached a plateau of $55 \%$ at 25 years of age (fig. 1). CFRD was found in $12.4 \%$ of the patients ( $11.2 \%$ of males and $13.7 \%$ of females); its prevalence increased with age, from almost absent in children aged $<10$ years to $22.7 \%$ in patients aged $\geqslant 30$ years.

\section{FEV1 characteristics}

FEV1 \% pred was negatively associated with age (fig 2): mean FEV1\% pred decreased from 91.2\% (95\% CI 90.4-91.9) in the 6-9-year-olds to 55.5\% (95\% CI 53.4-57.6) in the 40-44-year-olds (table 1). As shown in figure 2, the decline starts slowly, becomes sharper at age 12 years, continues until the age of 20 years and then stays fairly stable. In contrast, the number of patients (fig. 2) is quite uniform up to age 18 years, when it steadily decreases across the remaining years, although with a less steep decrease from the age of 30 years. Thus, we noticed a gap of roughly 6 years between the sharper decline in FEV1 and the subsequent decline in the number of patients.

Table 2 shows the differences in the estimated marginal means of FEV1 \% pred computed from the regression models: they represent the estimated effect of each factor on FEV1, after adjusting for age and country only and after adjusting for age, country, genotype, sex, pancreatic status, CFRD, $P$. aeruginosa infection and BMI. Genotype did not show a clinically relevant effect on FEV1: the highest estimated difference after adjusting for effect of age and country was between patients with the two severe alleles and patients with one severe and one mild allele, the difference in FEV1 being -10.9 percentage points (95\% CI -12.6- -9.1). This difference decreased to -3.8 percentage points (95\% CI -5.9--1.6) after adjusting for the other covariates.

Similarly, males had higher FEV1 \% pred values than females by 1.1 percentage points (95\% CI $0.3-1.8$ ) after adjusting for age and country effects, and this difference increased to 2.7 percentage points (95\% CI 1.8-3.6) after additional adjusting for genotype, BMI, pancreatic status, $P$. aeruginosa infection and presence of CFRD. Since sex did not reach the threshold of clinical relevance, it was not included in the final model.

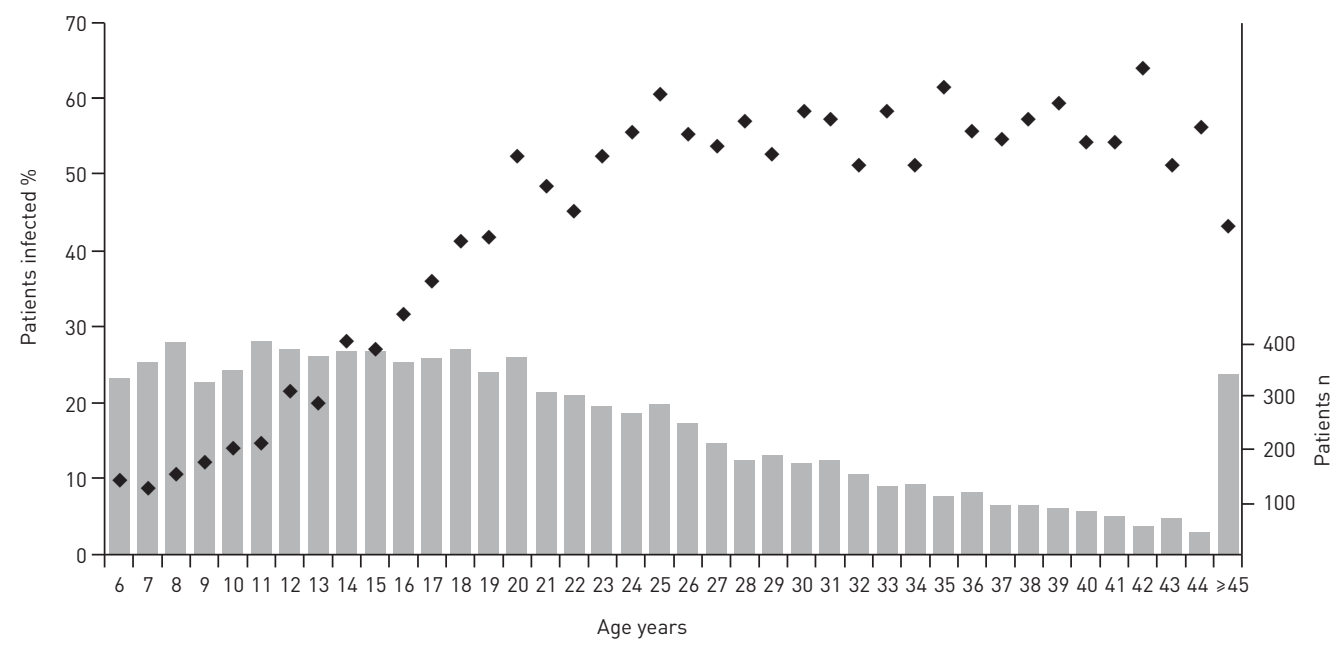

FIGURE 1 Prevalence of chronic Pseudomonas aeruginosa infection and total number of patients by age. 


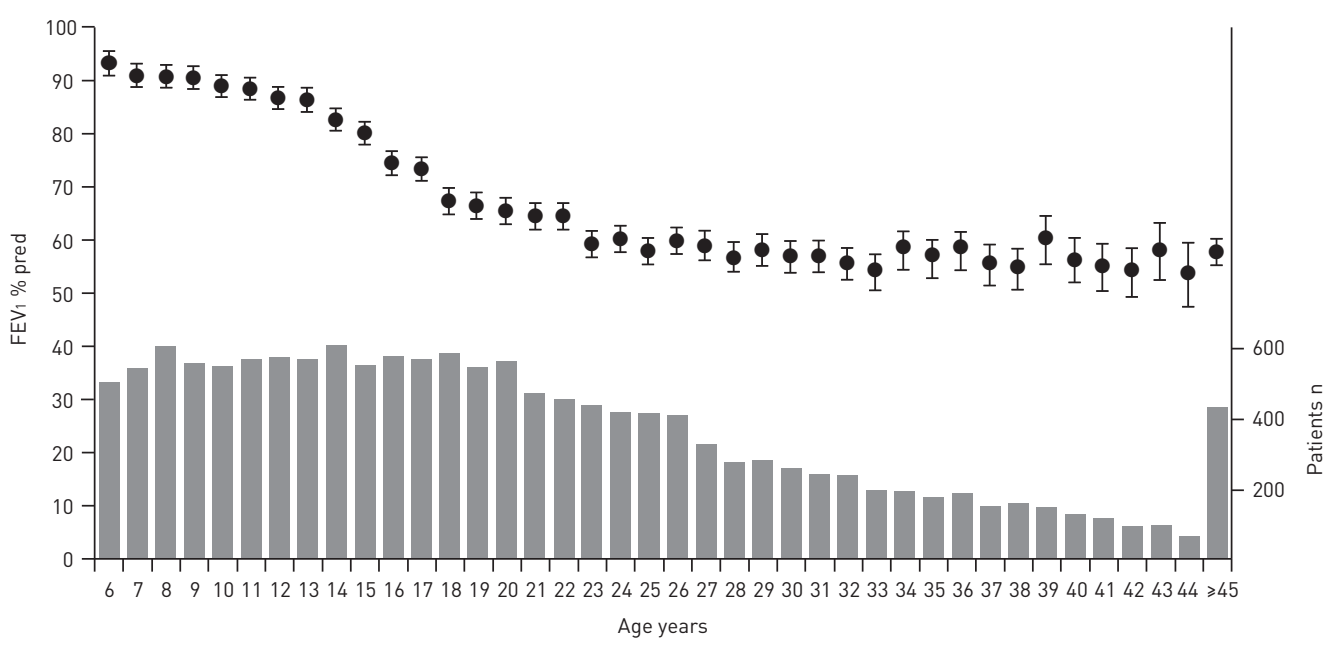

FIGURE 2 Forced expiratory volume in $1 \mathrm{~s}$ (FEV1) and number of patients by age. Data are presented as mean (95\% CI) unless otherwise stated. $\%$ pred: $\%$ predicted.

Pancreatic status was associated with FEV1 after adjusting for age and country $(\mathrm{p}<0.0001)$, but the difference between pancreatic-sufficient and -insufficient patients decreased from 12.9 percentage points (95\% CI 11.8-14.2) to 6.7 percentage points (95\% CI 5.2-8.2) after adjusting for sex, genotype, BMI, P. aeruginosa infection and CFRD.

Patients with CFRD showed FEV1 \% pred values 11.6 percentage points (95\% CI 10.4-12.8) lower than patients without CFRD, but this difference decreased to 8.0 percentage points (95\% CI 6.6-9.5) after additionally adjusting for sex, genotype, BMI, $P$. aeruginosa infection and pancreatic status.

TABLE 2 Differences in the estimated marginal means of forced expiratory volume in $1 \mathrm{~s}$ (FEV1) \% predicted computed from the linear regression models; representing the estimated effect of each factor on FEV1

Adjustment for age and country

\begin{tabular}{|c|c|c|c|c|}
\hline & & \\
\hline & Difference & $p$-value & Difference & p-value \\
\hline Genotype $^{\#}$ & & $<0.0001$ & & 0.0005 \\
\hline Severe/severe - severe/mild & $-10.9(-12.6--9.1)$ & & $-3.8(-5.9--1.6)$ & \\
\hline Severe/severe - unclassified & $-5.7(-6.5--4.8)$ & & $-2.8(-3.9--1.7)$ & \\
\hline Severe/mild - mild/mild & $3.8(-5.0-12.6)$ & & $4.5(-6.8-15.9)$ & \\
\hline Severe/mild - unclassified & $5.2(3.4-7.0)$ & & $1.0(-1.1-3.1)$ & \\
\hline Male - female & $1.1(0.3-1.8)$ & 0.0051 & $2.7(1.8-3.6)$ & $<0.0001$ \\
\hline \multicolumn{5}{|l|}{ Pancreatic status } \\
\hline Sufficient - insufficient & $12.9(11.8-14.2)$ & $<0.0001$ & $6.7(5.2-8.2)$ & $<0.0001$ \\
\hline \multicolumn{5}{|l|}{ CFRD } \\
\hline Yes - no & $-11.6(-12.8--10.4)$ & $<0.0001$ & $-8.0(-9.5--6.6)$ & $<0.0001$ \\
\hline \multicolumn{5}{|l|}{$\begin{array}{l}\text { Pseudomonas aeruginosa infec- } \\
\text { tion }\end{array}$} \\
\hline Above normal - poor & $38.5(32.8-44.1)$ & & $29.1(21.9-36.2)$ & \\
\hline Above normal - normal & $15.4(9.9-21.0)$ & & $7.4(0.5-14.4)$ & \\
\hline
\end{tabular}

Data are presented as mean (95\% CI), unless otherwise stated. CFRD: cystic fibrosis-related diabetes; BMI: body mass index. ${ }^{\text {: }}$ alleles classified according to McKoNE et al. [11]: severe (alleles that belong to either class I, II or III), mild (alleles that belong either to class IV or V) and unclassified lalleles that could not be classified into any of the previous classes); ": normal: -2-2 standard deviation scores (SDS); poor: $\leqslant-2$ SDS; above normal: $>2$ SDS. 
Chronic infection by P. aeruginosa was associated with FEV1 after adjusting for age, country, sex, genotype, BMI, pancreatic status, and presence of CFRD $(\mathrm{p}<0.0001)$. Infected patients showed mean values of FEV1 $\%$ pred 13.0 percentage points (95\% CI 12.0-14.0) lower than uninfected patients.

BMI was positively associated with FEV1 after adjusting for age, country, sex, genotype, P. aeruginosa infection, pancreatic status and presence of CFRD $(\mathrm{p}<0.0001)$. Patients showing a BMI within the normal range (i.e. between -2 and +2 SDS) had, on average, FEV1 \% pred 21.6 percentage points (95\% CI 20.0-23.2) higher than patients with poor BMI $(\leqslant-2$ SDS).

Based on these results, we selected age, BMI and chronic P. aeruginosa as potential modifiers of FEV1 in our final model due to their clinically relevant and their statistically significant effect. We also included pancreatic status and CFRD because, although their adjusted effect did not reach the clinically relevant threshold of 10 percentage points, from our data there was strong evidence of a non-negligible effect $(\sim 8$ percentage points). Age at diagnosis had a statistically significant effect on FEV1, after adjusting for all the previously described factors $(\mathrm{p}<0.0001)$, but it was not clinically relevant and therefore was not included in the final model: for each additional year of age at diagnosis, patients showed an increased FEV1 value of 0.16 percentage points ( $95 \%$ CI $0.10-0.22$ ). We carried out a sensitivity analysis of the selection of the covariates in the final model changing the minimal threshold of clinical relevance, and any threshold $>5$ percentage points difference in FEV1 (i.e. the variation that other authors claim exist in FEV1 due merely to different timing of FEV1 measurement) does not change the covariates that were selected in the final model, as can be seen from table 2 .

In this final model we tested for interaction between variables. We found a statistically significant interaction between $P$. aeruginosa infection and pancreatic status $(\mathrm{p}=0.012)$. After adjusting for country, age, BMI and presence of CFRD, pancreatic-sufficient patients not infected with $P$. aeruginosa had mean FEV1 values 16.5 percentage points higher than infected ones (95\% CI 13.8-19.2). Among pancreaticinsufficient patients, this difference reduced to 12.9 percentage points (95\% CI 11.8-13.9). Described from another perspective, pancreatic-sufficient patients not infected with $P$. aeruginosa had, on average, FEV1 values 9.0 percentage points higher than pancreatic-insufficient patients ( $95 \%$ CI 7.6-10.5). Among patients infected with $P$. aeruginosa, this difference was only 5.4 percentage points (95\% CI 2.9-7.9). There was also evidence of interaction between $P$. aeruginosa infection and CFRD $(\mathrm{p}=0.021)$. After adjusting for country, age, BMI and pancreatic status, the difference in FEV1 \% pred between infected and uninfected patients without CFRD was 13.8 percentage points (95\% CI 12.7-14.8) in favour of those not infected, whereas

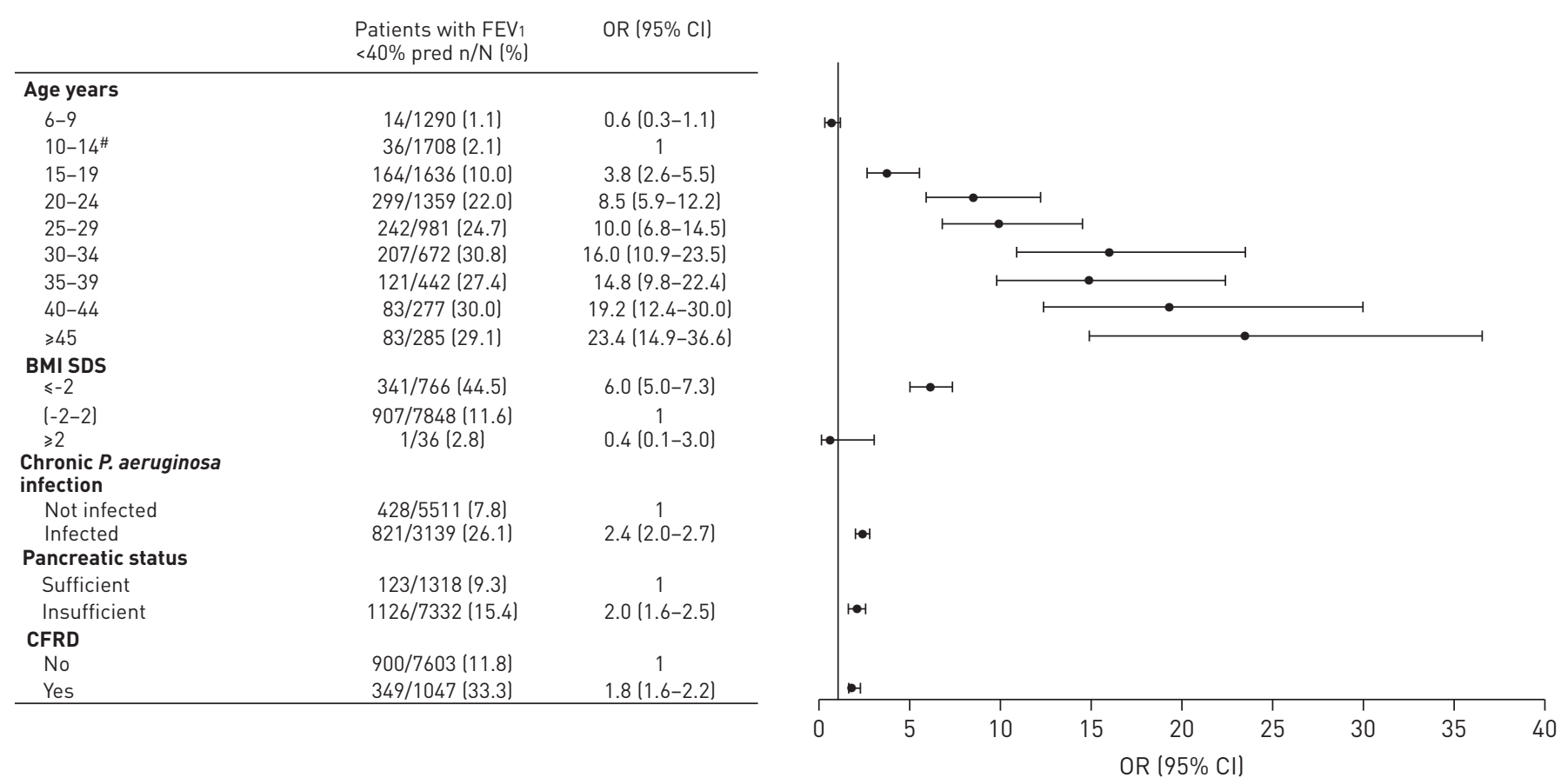

FIGURE 3 Multiple logistic model: effect of age, body mass index (BMI), chronic Pseudomonas aeruginosa infection, pancreatic status and cystic fibrosis-related diabetes (CFRD) on the odds of having forced expiratory volume in $1 \mathrm{~s}$ (FEV1) $<40 \%$ predicted. Estimates are controlled for country. Model estimated on 8650 patients from Austria, Belgium, Bulgaria, France, Greece, Italy, the Netherlands, Slovenia and UK. \% pred: \% predicted; SDS: standard deviation scores. ${ }^{\#}$ chosen as reference as most numerous group. 
among patients with CFRD the difference was 10.5 percentage points (95\% CI 7.9-13.1), in favour of those not infected.

While these differences are statistically significant, they are clinically negligible and therefore we did not include any interaction in the final model.

In order to express the magnitude of the effect of the risk factors identified in the previous analyses on severe lung disease we carried out a multiple logistic regression analysis (based on 8650 patients with information on all covariates). Severe lung disease was defined as having FEV $1<40 \%$ pred, a widely accepted clinical threshold. As shown in figure 3, the odds of having severe lung disease significantly increases with age (test for quadratic trend $\mathrm{p}<0.0001$ ). Of the other factors, BMI had the biggest impact on lung disease: patients with poor BMI (i.e. $\leqslant-2$ SDS) experienced a six-fold increased odds of severe lung disease (OR 6.0, 95\% CI 5.0-7.3) compared to patients with normal BMI (between -2 and +2 SDS). Chronic $P$. aeruginosa infection was the factor that had the second biggest impact: being infected increases the odds of severe lung disease by 2.4 (95\% CI 2.0-2.7). Furthermore, we estimated that pancreatic-insufficient patients experienced an odds of severe lung disease twice that of pancreatic-sufficient patients (95\% CI 1.6-2.5) and patients affected by CFRD had a 1.8-fold increased odds of severe lung disease compared to patients not affected by CFRD (OR 1.8, 95\% CI 1.6-2.2). When we inspected the variation of odds ratios between countries to check for interaction, we concluded that the odds ratios are similar across countries (results not shown).

\section{Discussion}

The heterogeneous European CF population showed a wide variability of factors potentially associated with FEV1. We aimed at identifying such factors after adjusting for potential confounders. In this population five factors were associated with poor pulmonary function in patients with $\mathrm{CF}$ aged $>5$ years. Three of the five factors are either partially preventable and/or potentially treatable (poor nutritional status, chronic $P$. aeruginosa infection and CFRD). The fourth, pancreatic function, indirectly represents the degree of CFTR dysfunction and it might be correctable in the future by therapies that increase the level of functional CFTR. The fifth factor, age, is challenging since it reflects the continuing exposure to additional deleterious factors that might be preventable or treatable with adherence to the current available therapies.

Low BMI was the strongest potentially preventable factor found in the current study of ECFSPR data. The odds of severe lung disease were six times higher for patients with very poor nutrition than patients with BMI within "normal" range, the highest odds ratio of all the preventable factors. Although a causal relationship cannot be proved by our study (severe lung disease by itself can cause poor nutrition through increased energy needs, reduced appetite and gastrointestinal involvement such as reflux and nausea), we showed that poor BMI was significantly associated with poor lung function, even after taking into account the effect of age, pancreatic status or infection with P. aeruginosa. The association between better nutritional status, better pulmonary status and survival has been already demonstrated [15], and aggressive intervention early in life aimed at growth and nutrition may positively affect pulmonary function [16]. However, the current study shows that, despite the available guidelines for prevention and correction of nutritional deficiencies in CF [17, 18], 9.5\% of the patients with CF in Europe still suffer from severe malnutrition (i.e. with a BMI $\leqslant-2$ SDS).

Chronic infection with P. aeruginosa was the second factor negatively associated with FEV1. This infection is well recognised as a cause of morbidity and mortality in CF [19]. In the current study, chronic P. aeruginosa infection was significantly associated with lower FEV1 after adjusting for age and other potential confounding factors and increased the likelihood of severe lung disease 2.4-fold. This association was also found by both VAnDeVanter et al. [1] and Konstan et al. [20]. The association seems to be stronger in our study, which could be attributed to the use by both authors of "Pseudomonas at least once" as their definition of infection, whereas we used "chronic Pseudomonas", which usually implies longer exposure and very often mucoid bacteria. Additionally, both authors investigated children/teenagers, who will have been exposed to the bacteria for a shorter time on average, especially in the chronic form, which would also tend to show a weaker association. However, it is noteworthy that even in young patients with only intermittent or early chronic infection the association is still significant. The importance of the association between $P$. aeruginosa infection and poor lung function is that although most $P$. aeruginosa infections are not acquired in hospital, acquisition by cross-infection can be prevented using infection-control measures. Furthermore, newly acquired infections can be eradicated in $>80 \%$ of the cases by antibiotic therapy immediately after the onset of P. aeruginosa infection [21]. Once chronically colonised, efforts should be directed towards suppression of the infection. Clinical studies have shown that inhaled antibiotics improved pulmonary function and reduced the rate of exacerbations in patients already infected with P. aeruginosa [22].

CFRD was found in the current study to be associated with lower lung function, including after controlling for age and BMI. It is the most frequent comorbidity diagnosed at present, occurring in up to $40 \%$ of adults, 
$25 \%$ of adolescents and $9 \%$ of children [23]. CFRD is associated with a rapid decline in lung function and increased risk of respiratory failure [24]. Given that CFRD is often clinically silent in the initial stages, it may be valuable to study the potential impact on FEV1 of annual CFRD screening for early diagnosis [17]. The current study confirms the association between CFRD and poor lung function. Since CFRD is currently not preventable, it should be diagnosed as early as possible. Treatment with insulin enhances nutritional status, temporarily improves pulmonary function and delays the decline in FEV1 [25].

Pancreatic status in CF is genetically determined and it is associated with CFTR genotype [26]. Pancreatic sufficiency in CF is associated with carrying at least one CFTR allele from class IV or V CFTR mutations that are associated with the presence of sufficient functional CFTR chloride channels over the apical membrane of the exocrine epithelial cells to maintain adequate pancreatic exocrine function. Compared to patients with pancreatic insufficiency, patients with pancreatic sufficiency are diagnosed later, have lower sweat chloride levels and better nutritional status $[20,26]$. The current study showed that pancreatic sufficiency is associated with higher lung function even after controlling for nutrition, infection with $P$. aeruginosa and CFRD. The importance of this finding is that patients with pancreatic sufficiency have better pulmonary function independent of other measured variables. A previous study by COREY et al. [27] showed that patients with pancreatic sufficiency had a lower rate of decline of FEV1. Therefore, for epidemiological studies and benchmark analysis, when comparing pulmonary function, control for pancreatic status is required, since the prevalence of pancreatic insufficiency may differ between cohorts. Pancreatic status (or the associated genotype) is not a preventable or treatable factor; however, emerging new therapies are under development that may recover some of the lost CFTR function, thereby shifting a "severe" CFTR mutation towards a "mild" partially functional mutation [6, 7], which is expected to be associated with better pulmonary function.

The ECFSPR data do not show evidence of an association between genotype and FEV1. This is in concordance with previous studies that showed variable lung disease in patients with CF regardless of their genotypes, raising the importance of the other variables previously indicated $[26,28]$.

Since pancreatic status and genotype are strongly related, an association between FEV1 and genotype could be expected as well, but since pancreatic status and genotype convey very similar information, once one covariate is present in the model, the other does not additionally explain much more variation of FEV1.

Although we did not find evidence in our data of the need to control for genotype for epidemiological and comparative studies, more detailed studies are needed to evaluate the importance of genotype on lung function.

In our study, as in other cross-sectional studies, FEV1 seems to be maintained in the advanced age groups (fig. 2). This is potentially a survivor effect: patients with the most severe lung disease are not included in cross-sectional studies because they died or underwent lung transplant, leaving an increased proportion of "less severely ill" patients in the eldest age groups.

In conclusion, the current study on a large patient population with CF shows the independent effect of nutrition, chronic P. aeruginosa infection and CFRD on FEV1, which is currently the gold standard measure of disease severity. Since these factors are to some degree preventable or are potentially treatable, this emphasises the importance of early identification of these modifiers of CF lung disease through adequate routine tests. Lowering the rates of new acquisition of $P$. aeruginosa together with methods to suppress its chronic infection and routine screening for CFRD should be additional therapeutic targets in CF care and should be used as benchmark comparisons for standards of care. Furthermore, for epidemiological and clinical studies when comparing groups of patients with $\mathrm{CF}$, it is important to standardise the patients groups not only according to age, but also according to pancreatic and nutritional status, chronic infection by $P$. aeruginosa and CFRD. Since this was an observational study based on registry data, there is the possibility of residual confounding effect of factors that were not measured in the registry.

\section{Acknowledgements}

We would like to thank the national representatives of the ECFSPR Steering group (full list accessible at www.ecfs.eu/ projects/ecfs-patient-registry/steering-committee), and in particular H. Jansen (Registre Belge de la Mucoviscidose/ Belgisch Mucoviscidose Register BMR-RBM, Belgium), I. Galeva (University Hospital “Alexandrovska”, Sofija, Bulgaria), S. Ravilly (Vaincre la Mucoviscidose, Paris, France), M. Stern (University Children’s Hospital, University of Tübingen, Tübingen, Germany), B.M. Assael (Cystic Fibrosis Center, Hospital of Verona, Verona, Italy) and C. Barreto (Dept of Child and Family, University Hospital de Santa Maria, Lisbon, Portugal).

\section{References}

1 VanDevanter DR, Wagener JS, Pasta DJ, et al. Pulmonary outcome prediction (POP) tools for cystic fibrosis patients. Pediatr Pulmonol 2010; 45: 1156-1166.

2 Rabin HR, Butler SM, Wohl ME, et al. Pulmonary exacerbations in cystic fibrosis. Pediatr Pulmonol 2004; 37: 400-406. 
3 Stern M, Wiedemann B, Wenzlaff P. From registry to quality management: the German Cystic Fibrosis Quality Assessment project 1995-2006. Eur Respir J 2008; 31: 29-35.

4 Knudsen PK, Olesen HV, Høiby N, et al. Differences in prevalence and treatment of Pseudomonas aeruginosa in cystic fibrosis centres in Denmark, Norway and Sweden. J Cyst Fibros 2009; 8: 135-142.

5 McCormick J, Sims EJ, Green MW, et al. Comparative analysis of Cystic Fibrosis Registry data from the UK with USA, France and Australasia. J Cyst Fibros 2005; 4: 115-122.

6 Accurso FJ, Rowe SM, Clancy JP, et al. Effect of VX-770 in persons with cystic fibrosis and the G551D-CFTR mutation. N Engl J Med 2010; 363: 1991-2003.

7 Kerem E, Hirawat S, Armoni S, et al. Effectiveness of PTC124 treatment of cystic fibrosis caused by nonsense mutations: a prospective phase II trial. Lancet 2008; 372: 719-727.

8 European Medicines Agency. Guideline on the clinical development of medicinal products for the treatment of cystic fibrosis. $\quad$ www.ema.europa.eu/ema/index.jsp?curl=pages/includes/document/document_detail.jsp?webContentId= WC500017055\&mid=WC0b01ac058009a3dc Date last accessed: August 9, 2012. Date last updated: May 12, 2011.

9 ECFS Patient Registry. ECFS Patient Registry Variables and Definitions. www.ecfs.eu/projects/ecfs-patient-registry/ Variables-Definitions Date last accessed: August 9, 2012. Date last updated: November 26, 2012.

10 Olesen H, Zolin A, Viviani L, et al. ECFS Patient Registry Report on 2007 Data. 2010. www.ecfs.eu/projects/ecfspatient-registry/annual-reports Date last accessed: August 9, 2012. Date last updated: April 21, 2010.

11 McKone E, Emerson SS, Edwards KL, et al. Effect of genotype on phenotype and mortality in cystic fibrosis: a retrospective cohort study. Lancet 2003; 361: 1671-1676.

12 Wang X, Dockery DW, Wypij D, et al. Pulmonary function between 6 and 18 years of age. Pediatr Pulmonol 1993; 15: 75-88.

13 Hankinson JL, Odencrantz JR, Fedan KB. Spirometric reference values from a sample of the general U.S. population. Am J Respir Crit Care Med 1999; 159: 179-187.

14 Kuczmarski RJ, Ogden CL, Guo SS, et al. 2000 CDC Growth Charts for the United States: methods and development. Vital Health Stat 11 2002; 246: 1-190.

15 Milla CE. Association of nutritional status and pulmonary function in children with cystic fibrosis. Curr Opin Pulm Med 2004; 10: 505-509.

16 Konstan MW, Butler SM, Wohl ME, et al. Growth and nutritional indexes in early life predict pulmonary function in cystic fibrosis. J Pediatr 2003; 142: 624-630.

17 Kerem E, Conway S, Elborn S, et al. Standards of care for patients with cystic fibrosis: a European consensus. J Cyst Fibros 2005; 4: 7-26.

18 Sinaasappel M, Stern M, Littlewood J, et al. Nutrition in patients with cystic fibrosis: a European Consensus. J Cyst Fibros 2002; 1: 51-75.

19 Emerson J, Rosenfeld M, McNamara S, et al. Pseudomonas aeruginosa and other predictors of mortality and morbidity in young children with cystic fibrosis. Pediatr Pulmonol 2002; 34: 91-100.

20 Konstan MW, Morgan WJ, Butler SM, et al. Risk factors for rate of decline in forced expiratory volume in one second in children and adolescents with cystic fibrosis. J Pediatr 2007; 151: 134-139.

21 Ratjen F, Munck A, Kho P, et al. Treatment of early Pseudomonas aeruginosa infection in patients with cystic fibrosis: the ELITE trial. Thorax 2010; 65: 286-291.

22 Ramsey BW, Pepe MS, Quan JM, et al. Intermittent administration of inhaled tobramycin in patients with cystic fibrosis. N Engl J Med 1999; 340: 23-30.

23 Moran A, Doherty L, Wang X, et al. Abnormal glucose metabolism in cystic fibrosis. J Pediatr 1998; 133: 10-17.

24 Milla CE, Warwick WJ, Moran A. Trends in pulmonary function in patients with cystic fibrosis correlate with the degree of glucose intolerance at baseline. Am J Respir Crit Care Med 2000; 162: 891-895.

25 Mohan K, Israel KL, Miller H, et al. Long-term effect of insulin treatment in cystic fibrosis-related diabetes. Respiration 2008; 76: 181-186.

26 Kerem E, Corey M, Kerem BS, et al. The relation between genotype and phenotype in cystic fibrosis - analysis of the most common mutation (delta F508). N Engl J Med 1990; 323: 1517-1522.

27 Corey M, Edwards L, Levison $\mathrm{H}$, et al. Longitudinal analysis of pulmonary function decline in patients with cystic fibrosis. J Pediatr 1997; 131: 809-814.

28 Burke W, Aitken ML, Chen SH, et al. Variable severity of pulmonary disease in adults with identical cystic fibrosis mutations. Chest 1992; 102: 506-509. 